\title{
Association Between CDKALI, HHEX, CDKN2A/2B and IGF2BP2 Gene Polymorphisms and Susceptibility to Type 2 Diabetes in Uttarakhand, India
}

This article was published in the following Dove Press journal:

Diabetes, Metabolic Syndrome and Obesity: Targets and Therapy

\author{
Amit $\mathrm{K}$ Verma ${ }^{\mathrm{I}, *}$ \\ Yamini Goyal ${ }^{1} *$ * \\ Deepti Bhatt ${ }^{1, *}$ \\ Mirza Masroor Ali Beg $2, *$ \\ Kapil Dev' \\ Mohammed A Alsahli ${ }^{3}$ \\ Arshad Husain Rahmani ${ }^{3}$ \\ 'Department of Biotechnology, Jamia \\ Millia Islamia, New Delhi, India; \\ ${ }^{2}$ Department of Biochemistry, Maulana \\ Azad Medical College, New Delhi, India; \\ ${ }^{3}$ Department of Medical Laboratories, \\ College of Applied Medical Sciences, \\ Qassim University, Buraydah, Saudi \\ Arabia \\ *These authors contributed equally to \\ this work
}

Introduction: Current study aimed to find the association of genes polymorphism of CDKAL1, HHEX, CDKN2A/2B, and IGF2BP2 with type 2 diabetes (T2DM) in the population of Uttarakhand.

Research Design and Methods: Overall 469 persons comprising 369 recently diagnosed T2DM cases and 100 healthy control were enrolled in the present study. The polymorphisms were analyzed through the PCR-RFLP technique.

Results: For the rs10440833 variant (CDKAL1), CC genotype's frequency was significantly high among T2DM subjects than controls and increase the T2DM risk (OR: 4.46, 95\% CI: $2.22-8.99, \mathrm{p}<0.0001)$. The $\mathrm{c}$ allele was significantly found to increase the T2DM risk (OR: 2.20, 95\% CI: $1.54-3.14, \mathrm{p}<0.001$ ). In the rs1111875 variant (HHEX), the difference of genotype frequencies among T2DM cases and control was statistically non-significant (p-0.138). We did not observe significant differences in allelic frequencies among T2DM cases and control (p-0.444). In the case of rs10811661 variant (CDKN2A/2B), frequency of both TC (OR: 3.16, 95\% CI: 1.84-5.42, p <0.0001) and TT (OR: 5.84, 95\% CI: 1.75-19.45, $\mathrm{p}-0.004)$ genotype were significantly higher in T2DM cases in comparison with control and significantly associated with higher T2DM risk. Compared to the $\mathrm{C}$ allele, a significant increase in T2DM risk was documented with the T allele (OR: $2.47,95 \%$ CI: $1.55-3.92$, $\mathrm{p}<0.001$ ). For rs4402960 variant (IGF2BP2), TT genotype contributed to increased T2DM risk (OR: $4.25,95 \%$ CI: 2.02-8.93, p-0.0001). T allele's frequency was significantly high in T2DM cases in comparison with healthy control. Except WHR, HDL-C, exercise, household chores, standing work more than 3 hours, and family history, significant differences were found between T2DM cases and healthy individuals in all other parameters.

Conclusion: Our study concluded a significant association of CDKAL1, CDKN2A/2B, and IGF2BP2 polymorphism with T2DM in the Uttarakhand population. For HHEX, the genotype and allelic frequencies difference between T2DM cases and control were statistically non-significant. However, a significant association of HHEX gene polymorphism with T2DM was observed only under the dominant model.

Keywords: type 2 diabetes, CDKAL1, CDKN2A/2B, IGF2BP2, HHEX, RFLP

\section{Introduction}

Diabetes prevalence has been continuously increasing day by day across the globe. According to the International Diabetes Federation (IDF) report, around 463 million had diabetes in 2019 and it will rise to 578 million by 2030 and 700 million by
Correspondence: Amit K Verma Department of Biotechnology, Srinivas Ramanujan Block, Mujeeb Bagh, Jamia Millia Islamia, Lab 4I3, Medical Biotechnology Lab, $4^{\text {th }}$ Floor, New Delhi I 10025 , India

Tel $+91-9027777719$

Email averma2@jmi.ac.in

Diabetes, Metabolic Syndrome and Obesity: Targets and Therapy 2021:14 23-36 
2045. About $90 \%$ of all diabetes cases belong to only type 2 diabetes (T2DM) and about 77 million people were living with T2DM in India in 2019. ${ }^{1}$ It is a chronic metabolic disorder and affected by genetic as well as environmental factors. ${ }^{2}$ Hyperglycaemia is characteristic of T2DM and it is related to the incapability of the human body to fully respond to insulin but over the period, production of insulin decreases. ${ }^{3,4} \mathrm{~T} 2 \mathrm{DM}$ is mostly diagnosed in middle-aged people but cases among adolescents, children have increased recently due to increasing levels of physical inactivity, obesity, and unhealthy diet. ${ }^{5}$ Excessive thirst, tiredness, frequent urination, blurred vision, slow healing wounds, numbness in hand and feet, persistent fungal infections in the skin are symptoms of T2DM. ${ }^{1}$ In some T2DM subjects, this disease is first diagnosed when chronic hyperglycaemic complications develop like the potential loss of vision, renal infection or failure, foot ulcer. $^{6-8}$ The etiology of T2DM is not fully known but there is a strong association with increasing age and obesity as well as with overweight and family history.

Several important risk factors involved in the development of T2DM are ethnicity or race, sedentary lifestyle, an unhealthy diet with high consumption of sugary drinks and low consumption of fiber-rich diet, prediabetes, smoking, depression, and medical conditions linked with insulin resistance like dyslipidemia, hypertension or polycystic ovary syndrome (PCOS). ${ }^{8}$ T2DM is characterized by hyperglycemia and chronic hyperglycemia can lead to several complications and is linked with long-term failure, dysfunction, and damage of several organs, mainly nerves, and blood vessels, kidneys, and eyes. ${ }^{9}$

Earlier, candidate gene analysis and linkage studies were the only techniques used to recognize genes which are involved in T2DM pathogenesis, but these techniques recognized only a small number of genes ie, Transcription Factor 7 Like 2 (TCF7L2), Calpain-10 (CAPN10), Peroxisome Proliferator-Activated Receptor Gamma (PPARG) and Potassium Inwardly Rectifying Channel Subfamily J Member 11 (KCNJ11). Advancement in genomic data and technology have helped the genome-wide association studies (GWAS) in recognizing the susceptible genes of T2DM. ${ }^{10}$ Numerous GWAS confirmed the association of susceptible genes with T2DM in or near CDK5 Regulatory Subunit Associated Protein 1-Like 1 (CDKAL1), PPARG, Solute Carrier Family 30 Member 8 (SLC30A8), TCF7L2, Melatonin Receptor 1b (MTNR1B), Potassium VoltageGated Channel, KQT-Like Subfamily, Member 1 (KCNQ1), Wolframin ER Transmembrane Glycoprotein
(WFS1), Hepatocyte Nuclear Factor 1 Homeobox B (HNF1B), Cyclin-Dependent Kinase Inhibitor 2A (CDKN2A/2B), Insulin-Like Growth Factor 2 mRNA Binding Protein 2 (IGF2BP2), and Juxtaposed with Another Zinc Finger Protein 1 (JAZF1) among Asian and European population. ${ }^{10-12}$ Chiefari et al studied the highmobility group A1 (HMGA1) gene variants in T2DM patients and controls in 3 populations of white European ancestry and found the association of functional HMGA1 variants in people with T2DM of European ancestry. ${ }^{13}$

Many biochemical parameters and sedentary life-style exhibited are associated with T2DM risk. ${ }^{14}$ Metabolic syndrome is a critical risk sign for stroke, diabetes, and cardiovascular diseases (CVD) and it is the incidence of obesity, hypertension, insulin resistance, and dyslipidemia together. ${ }^{15}$ Excessive alcohol and smoking amongst adults are very problematic due to the elevated risk of CVD associated with smoking, alcohol, and T2DM. ${ }^{16}$ T2DM subjects generally exhibit decreased physical action and a more sedentary lifestyle specifically by spending the most time sitting, by viewing television and mobile phone screen and this causes inequity in biochemical parameters for example cholesterol, Body mass index (BMI), blood pressure, triglycerides. ${ }^{17}$ These biochemical parameters are modifiable risk factors for T2DM.

CDKAL1 or cyclin-dependent kinase 5 regulatory subunit associated protein 1 gene is present at 6th chromosome at p22.3 location and CDK5 regulatory subunit associated protein 1 like 1 encoded by this gene. This plays an important part in beta-cell dysfunction and susceptibility to T2DM. ${ }^{18}$ HHEX or haematopoietically expressed homeobox gene encodes a protein that influences the Wnt pathway of signaling that is important for development as well as the growth of cells and it also regulates beta-cell function and maturation by activating HNF (hepatocyte nuclear factor) - 1a. ${ }^{19} \mathrm{CDKN} 2 \mathrm{~A} / 2 \mathrm{~B}$ or cyclin-dependent kinase inhibitor $2 \mathrm{~A} / 2 \mathrm{~B}$ gene present at 9th chromosome at position $\mathrm{p} 21$. Association of rs10811661 SNP in CDKN2A/2B with T2DM has been confirmed by various GWAS in different populations. ${ }^{20-22}$ Kong et al reported that the CDKN2A/2B gene may influence the risk of T2DM by reducing beta-cell proliferation and islet expression. ${ }^{23}$ IGF2BP2 or Insulin-like growth factor-2 mRNA binding protein-2 gene, highly manifested in islets of the pancreas and it belongs to the IGF-2 family of mRNA binding proteins and it plays a vital part in normal development and growth of embryo. ${ }^{24}$ Furthermore, IGF2BP2 has been reported to be linked 
with reduced secretion of insulin and it plays a considerable part in the pathogenesis of T2DM. ${ }^{25}$ Even with the coherent linkage in Europeans, the impacts of variants of these genes in different ethnic groups of the Indian population is not much clear. Due to the difference in ethnicity, lifestyle, and linkage disequilibrium (genetic background), it is essential to recognize the role of these genes in Indian ethnic groups. Our study focused on following four Single nucleotide polymorphisms (SNPs): CDKAL1 (rs10440833), HHEX (rs1111875), CDKN2A/ 2B (rs10811661) and IGF2BP2 (rs4402960). The purpose of the current study was to find the association of these four genes with T2DM and their association with clinicopathological parameters in the population of Uttarakhand, India.

\section{Materials and Methods Study Subjects}

Overall, 469 persons comprising 369 recently diagnosed T2DM cases and 100 healthy controls were enrolled in the present study. T2DM subjects and healthy individuals were chosen on basis of inclusion and exclusion conditions. The present study was permitted by IEC, JMI, Delhi, India and this study was conducted in accordance with the Declaration of Helsinki. Patient included in the present study were examined and the collection of samples were completed only after getting notified permission from involved people (T2DM cases/healthy control). Three milliliters of blood samples were withdrawn from all the study subjects and collected in plain vials. Fasting as well as postprandial samples collected in fluoride vials from all the 200 T2DM subjects and $1 \mathrm{~mL}$ blood in EDTA vials for Hbalc. All the criteria were followed, such as fasting blood glucose [glucose level $\geq 126 \mathrm{mg} / \mathrm{dL}$ ] and postprandial glucose [2-hour blood glucose $\geq 200 \mathrm{mg} / \mathrm{dL}$ ] were monitored for diagnosis of T2DM disease. Healthy control blood samples were also checked for the fasting blood glucose, postprandial glucose, and Hbalc level to confirm the free of T2DM.

All clinicopathological parameters like body mass index (BMI), age, fasting plasma glucose (FPG), fasting plasma insulin (FPI), postprandial glucose (PPG), systolic BP (mmHg), low-density lipoprotein (LDL), diastolic BP $(\mathrm{mmHg})$, high-density lipoprotein (HDL), cholesterol, triglycerides, and Haemoglobin $\mathrm{A}_{1 \mathrm{c}}(\mathrm{HbA} 1 \mathrm{c})$ were taken care as per standard criteria. A total of $2 \mathrm{~mL}$ of Blood samples were collected in EDTA coated vacutainer of
T2DM subjects and healthy persons. The non-diabetic (healthy persons) will be taken as a control in our study. (The samples were collected in the hospital by the expert medical professional according to guidelines provided by $\mathrm{ICMR} / \mathrm{GCP}$ ).

\section{Genotyping}

Isolation of DNA was done by the phenol-chloroform method from collected samples of blood of T2DM cases as well as healthy controls. Amplified DNA products were analyzed on $2 \%$ agarose gel and seen under UVtransilluminator. PCR was done using $7 \mu \mathrm{L}$ dd $_{2} \mathrm{O}, 1 \mu \mathrm{L}$ of each reverse and forward primer (total $2 \mu \mathrm{L}$ ), $1 \mu \mathrm{L}$ isolated DNA, and $10 \mu \mathrm{L}$ of master mix. PCR was done with initial denaturation temperature for $3 \mathrm{~min}$. at $94{ }^{\circ} \mathrm{C}$, followed by thirty-five cycles of denaturation for $30 \mathrm{sec}$. at $94{ }^{\circ} \mathrm{C}$, annealing (different temperature for each SNP) at $30 \mathrm{sec}$. and extension for $40 \mathrm{sec}$. at $72{ }^{\circ} \mathrm{C}$, then the final extension for $5 \mathrm{~min}$ at $72{ }^{\circ} \mathrm{C}$.

Amplified DNA to determine the genotype of CDKAL1 rs10440833 (C/A) by forward primer, 5' - AATT AATATTCCCCCCTGTATTTTAGT - 3' and reverse primer, 5' - GCTCATTGCTACATAATAACTGTAGAT - 3' using thermal cycler with annealing at $58^{\circ} \mathrm{C}$; HHEX rs1111875 (G/A) by forward primer, 5' - GGAAAAAA TTTGCACT GCCGTCT - 3' and reverse primer, 5' TGAGGCTGTAGATGAGATGACG-3' using thermal cycler with annealing at $60^{\circ} \mathrm{C}$; CDKN2A/2B rs10811661 (T/C) by forward primer, 5'- GAAGGATCCTTCTC - 3' and reverse primer, 5'- AGGATCGTAGTCTC - 3' using thermal cycler with annealing at $61^{\circ} \mathrm{C}$ and IGF2BP2 rs4402960 (T/G) by forward primer, 5' - AGACCAGCCT TGCAGCAATGTAGTG - 3' and reverse primer, 5' - CT AAAGCACTGAGAGAAACAGCCCT - 3' using thermal cycle with annealing at $59{ }^{\circ} \mathrm{C}$.

Restriction Fragment Length Polymorphism for each SNP was done by digesting $6 \mu \mathrm{L}$ PCR product with 2.5 units of restriction enzyme (NEB) and $1.5 \mu \mathrm{L}$ ddH2O overnight at $37{ }^{\circ} \mathrm{C}$. The restricted products were separated on 3\% agarose gel electrophoresis. Amplified DNA of CDKAL1 was digested by AciI and $\mathrm{A}$ allele and $\mathrm{C}$ allele were visualized as $157 \mathrm{bp}$ fragment and 121+36 bp bands, respectively. The amplified products of HHEX were digested by $\mathrm{XbaI}$ and $\mathrm{G}$ allele and $\mathrm{A}$ allele were visualized as $161 \mathrm{bp}$ fragment and $111+50 \mathrm{bp}$ bands, respectively. The amplified products of CDKN2A/2B were digested by BspHI and $\mathrm{C}$ allele and T allele were visualized as $232 \mathrm{bp}$ fragment and $164+68$ bp bands, respectively. The 
amplified products of IGF2BP2 were digested by MseI and $\mathrm{G}$ allele and $\mathrm{T}$ allele were visualized as $500 \mathrm{bp}$ fragment and $294+206$ bp bands, respectively.

\section{Statistical Analyses}

Frequencies of genotypes among T2DM subjects and healthy controls were found by applying the Chi-square test. Allelic frequencies were examined through the Hardy Weinberg Equilibrium equation. The associations between HHEX (rs1111875), CDKAL1 (rs10440833), CDKN2A/ 2B (rs10811661) and IGF2BP2 (rs4402960) genotype and T2DM risk were estimated by calculating OR with 95\% CI. Bonferroni test was applied to correction for multiple testing. The comparative assessment of clinicopathological parameters performed through a $t$-test for means equality. $\mathrm{P}$ values $<0.05$ considered significant. The relationship between genotype and investigated parameters was done by Analysis of Variance (ANOVA) One-Way ANOVA and Kruskal Wallis test.

\section{Results}

\section{Patient's Clinical Characteristics}

The clinicopathological and various other characteristics of the 369 T2DM subjects and 100 healthy controls are listed in Tables 1 and 2. T2DM subjects had a greater mean age compared to controls $(40.2 \pm 9.5$ vs $35.3 \pm 8.0$ years). Significant differences were found among T2DM subjects and healthy control in FPI, BMI, FPG, HbAlc, systolic BP, T - cholesterol, PPG, diastolic BP, LDL-C, triglycerides, urination, weight loss, blur vision, fatigue, appetite, slow wound healing, alcohol consumption, nonveg, smoking, consumption of fast food, consumption of sugary drinks, excessive calorie intake, normal walk, brisk walk $20 \mathrm{~min}$, prolonged sitting $3 \mathrm{hrs}$ work and leisure sitting tv mobile gross time $3 \mathrm{hrs}$ whereas, no significant difference was found between T2DM cases and controls with respect to WHR, HDL-C, exercise, household chores, standing work gross time $3 \mathrm{hrs}$ and family history.

\section{Association of CDKALI, HHEX, CDKN2A/2B and IGF2BP2 Gene Polymorphisms with T2DM}

The genotype and allele frequencies of CDKAL1, HHEX, CDKN2A/2B, IGF2BP2 polymorphism in T2DM cases and control are summarized in Table 3. For rs 10440833 variant (CDKAL1), 35.5\%, 26.6\%, 37.9\% and 46\%, 43\%, 11\% were the frequencies of genotypes $\mathrm{AA}, \mathrm{AC}$ and $\mathrm{CC}$ in $\mathrm{T} 2 \mathrm{DM}$ cases
Table I Clinicopathological Characteristics of T2DM Subjects and Healthy Controls

\begin{tabular}{|c|c|c|c|}
\hline Parameters & T2DM & Control & P-values \\
\hline $\mathrm{N}$ & 369 & 100 & - \\
\hline Age (Years) & $40.2 \pm 9.5$ & $35.3 \pm 8.0$ & $<0.001$ \\
\hline BMI $(\mathrm{kg} / \mathrm{m} 2)$ & $28.5 \pm 5.2$ & $24.8 \pm 2.3$ & $<0.001$ \\
\hline WHR & $0.9 \pm 0.1$ & $0.9 \pm 0.0$ & 0.720 \\
\hline FPG (mg/dl) & $|35| \pm 26.0$. & $90.2 \pm 7.1$ & $<0.001$ \\
\hline PPG (mg/dl) & $204.2 \pm 35.9$ & $136.2 \pm 4.5$ & $<0.001$ \\
\hline HbAlc (\%) & $7.1 \pm 1.1$ & $5.7 \pm 0.5$ & $<0.001$ \\
\hline FPI (mIU/L) & $9.6 \pm 1.4$ & $8.7 \pm 0.7$ & $<0.001$ \\
\hline Systolic BP (mmHg) & $145.1 \pm 17.3$ & $106.1 \pm 10.4$ & $<0.001$ \\
\hline Diastolic BP (mmHg) & $102.4 \pm 15.8$ & $75.9 \pm 10.9$ & $<0.001$ \\
\hline T-Cholesterol (mg/dl) & $245.5 \pm 15.2$ & $152.6 \pm 18.8$ & $<0.001$ \\
\hline $\mathrm{HDL}-\mathrm{C}(\mathrm{mg} / \mathrm{dl})$ & $46.8 \pm 11.1$ & $46.2 \pm 8.7$ & 0.621 \\
\hline $\mathrm{LDL}-\mathrm{C}(\mathrm{mg} / \mathrm{dl})$ & $192.3 \pm 29.1$ & $106.4 \pm 19.9$ & $<0.001$ \\
\hline Triglycerides (mg/dl) & $357.9 \pm 99.1$ & $14 \mid .0 \pm 5.5$ & $<0.001$ \\
\hline
\end{tabular}

Notes: P-value $<0.05$ considered significant, Data presented as Mean \pm SD. Abbreviations: $B M I$, body mass index; $\mathrm{HbAlc}$, haemoglobin $\mathrm{A}_{\mathrm{Ic}}$ PPG, postprandial plasma glucose; HDL, high-density lipoprotein; WHR, waist-to-hip ratio; FPI, fasting plasma insulin; LDL, low-density lipoprotein; FPG, fasting plasma glucose.

and healthy control, respectively. Through reference to the AA genotype, the frequency of CC genotype was significantly higher among T2DM subjects in comparison with control and increase the T2DM risk (OR: 4.46, 95\% CI: $2.22-8.99, \mathrm{p}<0.0001)$. The statistical analysis of observed genotypic frequencies for CDKAL1 (rs10440833) showed a significant association $(\mathrm{p}<0.0001)$. C allele frequency was significantly higher among T2DM cases in comparison with controls ( $51.22 \%$ vs $32.5 \%$, respectively). Compared to the A allele, the $\mathrm{C}$ allele was significantly found to increase the risk of T2DM (OR: 2.20, 95\% CI: 1.54-3.14, p $<0.001$ ). A significant relation was observed among CDKAL1 polymorphism and T2DM risk under the recessive model but not under the dominant model.

In the case of rs1111875 variant (HHEX), the frequencies of genotypes GG, AG, and AA among T2DM subjects and healthy controls were $51.22 \%, 31.44 \%$, and $17.34 \%$, and $44 \%, 42 \%$, and $14 \%$, respectively. The genotype frequencies difference between T2DM cases and control were statistically non-significant (p-0.138). The A allele frequencies were $33.06 \%$ and $35 \%$ in T2DM cases and controls, respectively. We did not observe significant 
Table 2 Characteristics of T2DM Subjects and Controls

\begin{tabular}{|c|c|c|c|}
\hline Parameters & $\begin{array}{l}\text { T2DM } \\
\mathbf{N}(\%)\end{array}$ & $\begin{array}{l}\text { Control } \\
\text { N (\%) }\end{array}$ & P-values \\
\hline \multicolumn{4}{|l|}{ Urination } \\
\hline No & $198(53.7)$ & $94(94.0)$ & $<0.001$ \\
\hline Yes & I7I (46.3) & $6(6.0)$ & \\
\hline \multicolumn{4}{|l|}{ Weight loss } \\
\hline No & $224(60.7)$ & $82(82.0)$ & $<0.001$ \\
\hline Yes & $145(39.3)$ & $18(18.0)$ & \\
\hline \multicolumn{4}{|l|}{ Fatigue } \\
\hline No & $193(52.3)$ & $84(84.0)$ & $<0.001$ \\
\hline Yes & $176(47.7)$ & $16(16.0)$ & \\
\hline \multicolumn{4}{|l|}{ Slow wound healing } \\
\hline No & $197(53.4)$ & $97(97.0)$ & $<0.001$ \\
\hline Yes & $172(46.6)$ & $3(3.0)$ & \\
\hline \multicolumn{4}{|l|}{ Blur vision } \\
\hline No & $202(54.7)$ & $82(82.0)$ & $<0.001$ \\
\hline Yes & $167(45.3)$ & $18(18.0)$ & \\
\hline \multicolumn{4}{|l|}{ Appetite } \\
\hline No & $181(49.1)$ & $85(85.0)$ & $<0.001$ \\
\hline Yes & $188(50.9)$ & $15(15.0)$ & \\
\hline \multicolumn{4}{|l|}{ Smoking } \\
\hline Never & $197(53.4)$ & $85(85.0)$ & $<0.001$ \\
\hline Former & $20(5.4)$ & $0(0.0)$ & \\
\hline Current & $152(4 \mid .2)$ & $15(15.0)$ & \\
\hline \multicolumn{4}{|l|}{ Alcohol consumption } \\
\hline Never & $194(52.6)$ & $90(90.0)$ & $<0.001$ \\
\hline Former & $17(4.6)$ & $0(0.0)$ & \\
\hline Current & $158(42.8)$ & $10(10.0)$ & \\
\hline \multicolumn{4}{|l|}{ Non-veg } \\
\hline No & $95(25.7)$ & $62(62.0)$ & $<0.001$ \\
\hline Normal & $172(46.6)$ & $34(34.0)$ & \\
\hline Low & $0(0.0)$ & $0(0.0)$ & \\
\hline High & $102(27.6)$ & $4(4.0)$ & \\
\hline \multicolumn{4}{|l|}{ Consumption of fast food } \\
\hline No & $0(0.0)$ & $20(20.0)$ & $<0.001$ \\
\hline Normal & $152(4 \mid .2)$ & $39(39.0)$ & \\
\hline Low & $98(26.6)$ & $36(36.0)$ & \\
\hline High & $119(32.2)$ & $5(5.0)$ & \\
\hline \multicolumn{4}{|l|}{$\begin{array}{l}\text { Consumption of sugary } \\
\text { drinks }\end{array}$} \\
\hline No & $0(0.0)$ & $0(0.0)$ & 0.001 \\
\hline Normal & $0(0.0)$ & $0(0.0)$ & \\
\hline Low & $113(30.6)$ & $4 \mid(4 I .0)$ & \\
\hline High & $78(2 I . I)$ & $5(5.0)$ & \\
\hline Medium & $178(48.2)$ & $54(54.0)$ & \\
\hline
\end{tabular}

(Continued)
Table 2 (Continued).

\begin{tabular}{|c|c|c|c|}
\hline Parameters & $\begin{array}{l}\text { T2DM } \\
\text { N (\%) }\end{array}$ & $\begin{array}{l}\text { Control } \\
\text { N (\%) }\end{array}$ & P-values \\
\hline $\begin{array}{l}\text { Excessive calorie intake } \\
\text { No } \\
\text { Normal } \\
\text { Low } \\
\text { High } \\
\text { Medium }\end{array}$ & $\begin{array}{l}0(0.0) \\
100(27.1) \\
113(30.6) \\
101(27.4) \\
55(14.9)\end{array}$ & $\begin{array}{l}0(0.0) \\
50(50.0) \\
36(36.0) \\
14(14.0) \\
0(0.0)\end{array}$ & $<0.001$ \\
\hline $\begin{array}{c}\text { Exercise } \\
\text { No } \\
\text { Yes }\end{array}$ & $\begin{array}{l}\text { I89 (5I.2) } \\
\text { I80 (48.8) }\end{array}$ & $\begin{array}{l}48(48.0) \\
52(52.0)\end{array}$ & 0.568 \\
\hline $\begin{array}{l}\text { Normal walk } \\
\text { No } \\
\text { Yes }\end{array}$ & $\begin{array}{l}186(50.4) \\
\text { I83 (49.6) }\end{array}$ & $\begin{array}{l}17(17.0) \\
83(83.0)\end{array}$ & $<0.001$ \\
\hline $\begin{array}{l}\text { Household chores } \\
\text { No } \\
\text { Yes }\end{array}$ & $\begin{array}{l}\text { I48 (46.4) } \\
\text { I7I (53.6) }\end{array}$ & $\begin{array}{l}39(39.0) \\
61(61.0)\end{array}$ & 0.194 \\
\hline $\begin{array}{l}\text { Brisk walk 20mints } \\
\text { No } \\
\text { Yes }\end{array}$ & $\begin{array}{l}226(6 \mid .2) \\
143(38.8)\end{array}$ & $\begin{array}{l}24(24.0) \\
76(76.0)\end{array}$ & $<0.001$ \\
\hline $\begin{array}{l}\text { Prolonged sitting } 3 \mathrm{hrs} \\
\text { work } \\
\text { No } \\
\text { Yes }\end{array}$ & $\begin{array}{l}15 \mid(40.9) \\
218(59.1)\end{array}$ & $\begin{array}{l}54(54.0) \\
46(46.0)\end{array}$ & 0.019 \\
\hline $\begin{array}{l}\text { Leisure sitting tv mobile } \\
\text { gross time } 3 \mathrm{hrs} \\
\text { No } \\
\text { Yes }\end{array}$ & $\begin{array}{l}6 I(16.5) \\
308(83.5)\end{array}$ & $\begin{array}{l}37(37.0) \\
63(63.0)\end{array}$ & $<0.001$ \\
\hline $\begin{array}{l}\text { Standing work gross time } \\
\text { 3hrs } \\
\text { No } \\
\text { Yes }\end{array}$ & $\begin{array}{l}|4|(38.2) \\
228(6 \mid .8)\end{array}$ & $\begin{array}{l}48(48.0) \\
52(52.0)\end{array}$ & 0.077 \\
\hline $\begin{array}{l}\text { Family History } \\
\text { No } \\
\text { Yes }\end{array}$ & $\begin{array}{l}205(55.6) \\
164(44.4)\end{array}$ & $\begin{array}{l}61(61.0) \\
39(39.0)\end{array}$ & 0.330 \\
\hline
\end{tabular}

Notes: Data presented as N (\%), P-value $<0.05$ considered significant.

differences in allelic frequencies among T2DM cases and healthy control ( $p$ - 0.444). We observed a significant association of HHEX gene polymorphism with T2DM only under the dominant model. However, no such association was observed under the recessive model.

For rs10811661 variant (CDKN2A/2B), the distribution of CC, TC and TT genotypes were $48.8 \%, 40.1 \%$, and $11.1 \%$ in $\mathrm{T} 2 \mathrm{DM}$ cases and $77 \%, 20 \%$, and $3 \%$ in controls, 
Table 3 Genotype, Allele Distribution, and Association Analysis of CDKALI, HHEX, CDKN2A/2B, and IGF2BP2 Gene Polymorphisms with T2DM Under Different Genetic Models

\begin{tabular}{|c|c|c|c|c|c|}
\hline Gene/SNP ID & Genotype/Allele & Cases $(n=369)$ & Control $(n=100)$ & Odds Ratio $(95 \% \mathrm{CI})$ & P-value \\
\hline \multirow[t]{8}{*}{ CDKALI rs 10440833} & AA & $|3|(35.5 \%)$ & $46(46 \%)$ & Ref & Ref \\
\hline & $A C$ & $98(26.6 \%)$ & $43(43 \%)$ & $0.80(0.48-1.30)$ & 0.374 \\
\hline & $\mathrm{CC}$ & 140 (37.9\%) & II (II\%) & $4.46(2.22-8.99)$ & $<0.0001 *$ \\
\hline & & $P$ value $<0.000$ & & \multirow{3}{*}{$2.20(1.54-3.14)$} & \multirow{3}{*}{$<0.001 *$} \\
\hline & A & $360(48.78 \%)$ & $135(67.5 \%)$ & & \\
\hline & C & 378 (5I.22\%) & 65 (32.5\%) & & \\
\hline & Recessive model & & & $6.40(3.16-13.00)$ & $<0.00 I^{*}$ \\
\hline & Dominant model & & & $0.77(0.48-1.25)$ & 0.290 \\
\hline \multirow[t]{9}{*}{ HHEX rsIIII 875} & & & & & \\
\hline & GG & $189(51.22 \%)$ & 44 (44\%) & Ref & Ref \\
\hline & AG & 116 (3I.44\%) & $42(42 \%)$ & $0.64(0.39-1.04)$ & 0.072 \\
\hline & AA & $64(17.34 \%)$ & 14 (14\%) & $1.06(0.54-2.06)$ & 0.854 \\
\hline & & $P$ value -0.138 & & \multirow{3}{*}{$0.87(0.6 \mathrm{I}-\mathrm{I} .24)$} & \multirow{3}{*}{0.444} \\
\hline & G & 494 (66.94\%) & $130(65 \%)$ & & \\
\hline & A & $244(33.06 \%)$ & $70(35 \%)$ & & \\
\hline & Recessive model & & & $1.57(0.80-3.10)$ & 0.191 \\
\hline & Dominant model & & & $1.65(1.01-2.68)$ & $0.044^{*}$ \\
\hline \multirow[t]{8}{*}{ CDKN2A/2B rs 10811661} & $\mathrm{CC}$ & $180(48.8 \%)$ & 77 (77\%) & Ref & Ref \\
\hline & $\mathrm{TC}$ & $148(40.1 \%)$ & $20(20 \%)$ & $3.16(1.84-5.42)$ & $<0.0001 *$ \\
\hline & TT & $41(11.1 \%)$ & $3(3 \%)$ & $5.84(1.75-19.45)$ & $0.004^{*}$ \\
\hline & & $P$ value $<0.000$ & & \multirow{3}{*}{$2.47(1.55-3.92)$} & \multirow{3}{*}{$<0.00 I^{*}$} \\
\hline & C & 508 (68.83\%) & $174(87 \%)$ & & \\
\hline & $\mathrm{T}$ & $230(31.17 \%)$ & $26(13 \%)$ & & \\
\hline & Recessive model & & & $3.30(0.96-11.33)$ & 0.058 \\
\hline & Dominant model & & & $0.37(0.22-0.63)$ & $<0.00 I^{*}$ \\
\hline \multirow[t]{8}{*}{ IGF2BP2 rs 4402960} & GG & $162(43.9 \%)$ & 62 (62\%) & Ref & Ref \\
\hline & GT & $107(29 \%)$ & $29(29 \%)$ & $\mathrm{I} .4 \mathrm{I}(0.85-2.33)$ & 0.179 \\
\hline & TT & $100(27.1 \%)$ & $9(9 \%)$ & $4.25(2.02-8.93)$ & $0.0001 *$ \\
\hline & & $P$ value -0.0002 & & \multirow{3}{*}{$2.50(1.70-3.68)$} & \multirow{3}{*}{$<0.00 I^{*}$} \\
\hline & G & $431(58.4 \%)$ & 153 (76.5\%) & & \\
\hline & $\mathrm{T}$ & 307 (41.6\%) & 47 (23.5\%) & & \\
\hline & Recessive model & & & $6.16(2.82-13.44)$ & $<0.00 I^{*}$ \\
\hline & Dominant model & & & $0.58(0.35-0.94)$ & $0.026 *$ \\
\hline
\end{tabular}

Notes: P-value was adjusted for $\mathrm{BMI}$ and age, $* \mathrm{P}$-value $<0.05$ considered significant. Data presented as odds ratio $(95 \% \mathrm{Cl})$. 
Table 4 Clinical Characteristics Based on CDKALI Genotypes in T2DM Subjects and Controls

\begin{tabular}{|c|c|c|c|c|c|c|c|c|c|}
\hline \multirow[t]{2}{*}{ Parameters } & \multicolumn{3}{|l|}{$C C(N=|5|)$} & \multicolumn{3}{|l|}{$A C(N=141)$} & \multicolumn{3}{|l|}{$A A(N=177)$} \\
\hline & Disease & Control & P-values & Disease & Control & P-values & Disease & Control & P-values \\
\hline Age (Years) & $41.2 \pm 8.9$ & $35.7 \pm 7.5$ & 0.059 & $40.6 \pm 10.0$ & $35.0 \pm 8.0$ & 0.001 & $38.9 \pm 9.5$ & $35.5 \pm 8.2$ & 0.029 \\
\hline BMI (kg/m2) & $27.4 \pm 5.6$ & $25.7 \pm 2.4$ & 0.240 & $30.9 \pm 4.7$ & $25.0 \pm 2.5$ & $<0.001$ & $27.7 \pm 4.6$ & $24.4 \pm 2.1$ & $<0.001$ \\
\hline WHR & $0.9 \pm 0.0$ & $0.9 \pm 0.0$ & 0.037 & $0.9 \pm 0.1$ & $0.9 \pm 0.0$ & 0.387 & $0.9 \pm 0.1$ & $0.9 \pm 0.1$ & 0.409 \\
\hline FPG (mg/dl) & $136.6 \pm 25.1$ & $90.1 \pm 8.3$ & $<0.001$ & $134.2 \pm 28.0$ & $88.9 \pm 7.5$ & $<0.001$ & $134.1 \pm 25.5$ & $91.5 \pm 6.4$ & $<0.001$ \\
\hline PPG (mg/dl) & $208.8 \pm 36.8$ & $137.5 \pm 2.0$ & $<0.001$ & $201.7 \pm 38.7$ & $136.2 \pm 5.6$ & $<0.001$ & $201.4 \pm 32.3$ & $135.9 \pm 3.8$ & $<0.001$ \\
\hline $\mathrm{HbAlc}$ & $7.1 \pm 1.1$ & $5.6 \pm 0.7$ & $<0.001$ & $7.1 \pm 1.1$ & $5.8 \pm 0.4$ & $<0.001$ & $7.2 \pm 1.2$ & $5.7 \pm 0.6$ & $<0.001$ \\
\hline FPI & $9.5 \pm 1.4$ & $8.8 \pm 0.8$ & 0.074 & $9.6 \pm 1.5$ & $8.6 \pm 0.6$ & $<0.001$ & $9.6 \pm 1.3$ & $8.7 \pm 0.8$ & $<0.001$ \\
\hline Systolic BP (mmHg) & $144.6 \pm 18.9$ & $103.1 \pm 11.0$ & $<0.001$ & $144.7 \pm 18.2$ & $107.6 \pm 11.1$ & $<0.001$ & $145.9 \pm 14.9$ & $105.3 \pm 9.6$ & $<0.001$ \\
\hline Diastolic BP (mmHg) & $101.4 \pm 14.7$ & $74.3 \pm 13.4$ & $<0.001$ & $102.5 \pm 16.4$ & $76.1 \pm 11.2$ & $<0.001$ & $103.4 \pm 16.5$ & $76.0 \pm 10.2$ & $<0.001$ \\
\hline T - Cholesterol (mg/dl) & $247.1 \pm 14.2$ & $153.2 \pm 17.4$ & $<0.001$ & $245.0 \pm 16.4$ & $154.1 \pm 19.3$ & $<0.001$ & $244.2 \pm 15.2$ & $151.1 \pm 19.0$ & $<0.001$ \\
\hline $\mathrm{HDL}-\mathrm{C}(\mathrm{mg} / \mathrm{dl})$ & $46.2 \pm 11.4$ & $47.7 \pm 7.7$ & 0.644 & $46.7 \pm 11.0$ & $46.7 \pm 8.3$ & 0.981 & $47.5 \pm 11.0$ & $45.4 \pm 9.3$ & 0.245 \\
\hline $\mathrm{LDL}-\mathrm{C}(\mathrm{mg} / \mathrm{dl})$ & $194.8 \pm 28.6$ & $105.5 \pm 21.3$ & $<0.001$ & $191.3 \pm 30.2$ & $107.4 \pm 19.1$ & $<0.001$ & $190.3 \pm 28.9$ & $105.7 \pm 20.8$ & $<0.001$ \\
\hline Triglycerides (mg/dl) & $368.6 \pm 98.4$ & $141.3 \pm 5.7$ & $<0.001$ & $351.4 \pm 95.7$ & $141.9 \pm 5.8$ & $<0.001$ & $351.4 \pm 102.1$ & $140.0 \pm 5.1$ & $<0.001$ \\
\hline
\end{tabular}

Notes: P-value $<0.05$ considered significant, P-values are obtained from Univariate Analysis of Variance, Bonferroni correction has been applied, Data presented as Mean \pm SD. Abbreviations: BMI, body mass index; HbAlc, haemoglobin $A_{I c ;}$ PPG, postprandial plasma glucose; HDL, high-density lipoprotein; WHR, waist-to-hip ratio; FPI, fasting plasma insulin; LDL, low-density lipoprotein; FPG, fasting plasma glucose.

respectively. Regarding CC genotype, frequency of both TC and TT genotype was significantly high in cases than control and significantly associated with higher T2DM risk (OR: $3.16,95 \% \mathrm{CI}: 1.84-5.42, \mathrm{p}<0.0001$ for $\mathrm{TC}$; and OR: $5.84,95 \%$ CI: $1.75-19.45$, p - 0.004 for TT, respectively). The statistical analysis of observed genotypic frequencies for $\mathrm{CDKN} 2 \mathrm{~A} / 2 \mathrm{~B} \quad(\mathrm{rs} 10811661)$ showed a significant association $(\mathrm{p}<0.0001)$. Similarly, the frequency of the CDKN2A/2B T allele was significantly high in $\mathrm{T} 2 \mathrm{DM}$ cases than in control $(31.17 \%$ vs $13 \%)$. Compared to the $\mathrm{C}$ allele, a significant increase in T2DM risk was documented with the $\mathrm{T}$ allele (OR: $2.47,95 \% \mathrm{CI}$ : $1.55-3.92, \mathrm{p}<0.001)$. We detected a significant association of the $\mathrm{CDKN} 2 \mathrm{~A} / 2 \mathrm{~B}$ gene $(\mathrm{C} / \mathrm{T})$ polymorphism with T2DM under the dominant model but no significant association under the recessive model.

For rs4402960 variant (IGF2BP2), 43.9\%, 29\%, 27.1\% and $62 \%, 29 \%, 9 \%$ were the frequencies of genotypes GG, GT and TT among T2DM cases and healthy controls respectively. When the GG genotype was set as the reference, the TT genotype contributed to increased T2DM risk (OR: 4.25, 95\% CI: 2.02-8.93, p - 0.0001). The statistical analysis of observed genotypic frequencies for IGF2BP2 (rs4402960) showed a significant association ( $p-0.0002)$.
T allele's frequency was significantly high in T2DM cases in comparison with healthy control $(41.6 \%$ vs $23.5 \%)$. Regarding allelic frequencies of the $\mathrm{T}$ allele as compared to the $\mathrm{G}$ allele, we found a significant association with increased T2DM risk (OR: 2.50, 95\% CI: 1.70-3.68, $\mathrm{p}<0.001$ ). Likewise, a significant relationship between IGF2BP2 gene $\mathrm{G}>\mathrm{T}$ polymorphism and T2DM risk was observed under the recessive and dominant model.

\section{Relationship of CDKALI, HHEX, CDKN2A/2B and IGF2BP2 Polymorphisms with Clinical Characteristics of T2DM Cases and Healthy Controls}

Association of CDKAL1 genotypes and investigated clinical characteristics with T2DM are shown in Table 4. Subjects carrying CC genotype had significantly higher PPG, systolic BP, WHR, T - Cholesterol, FPG, diastolic $\mathrm{BP}, \mathrm{HbA1c}$, LDL-C, and triglycerides compared to controls, whereas did not find a significant difference with respect to age, BMI, FPI, and HDL-C. Subjects with AC and AA genotype had significantly higher age, BMI, FPG, PPG, HbA1c, FPI, systolic BP, T-Cholesterol, diastolic BP, 
Table 5 Clinical Characteristics Based on HHEX Genotypes in T2DM Subjects and Controls

\begin{tabular}{|c|c|c|c|c|c|c|c|c|c|}
\hline \multirow[t]{2}{*}{ Parameters } & \multicolumn{3}{|l|}{ GG $(\mathbf{N}=233)$} & \multicolumn{3}{|l|}{ AG $(N=\mid 58)$} & \multicolumn{3}{|l|}{ AA $(\mathrm{N}=78)$} \\
\hline & Disease & Control & P-values & Disease & Control & P-values & Disease & Control & P-values \\
\hline Age (Years) & $40.2 \pm 9.2$ & $34.9 \pm 7.3$ & 0.001 & $40.6 \pm 9.5$ & $34.8 \pm 8.3$ & 0.001 & $39.5 \pm 10.3$ & $37.8 \pm 8.9$ & 0.516 \\
\hline BMI (kg/m2) & $27.8 \pm 5.1$ & $24.8 \pm 2.6$ & $<0.001$ & $30.2 \pm 5.1$ & $25.0 \pm 2.0$ & $<0.001$ & $27.4 \pm 5.2$ & $24.4 \pm 2.6$ & 0.033 \\
\hline WHR & $0.9 \pm 0.1$ & $0.9 \pm 0.1$ & 0.808 & $0.9 \pm 0.1$ & $0.9 \pm 0.0$ & 0.310 & $0.9 \pm 0.1$ & $0.9 \pm 0.1$ & 0.693 \\
\hline FPG (mg/dl) & $135.2 \pm 27.1$ & $90.3 \pm 7.2$ & $<0.001$ & $133.5 \pm 28.4$ & $89.2 \pm 7.4$ & $<0.001$ & $137.3 \pm 17.0$ & $92.8 \pm 5.7$ & $<0.001$ \\
\hline PPG (mg/dl) & $203.2 \pm 36.0$ & $135.7 \pm 5.2$ & $<0.001$ & $201.6 \pm 39.9$ & $136.8 \pm 3.9$ & $<0.001$ & $211.6 \pm 27.0$ & $136.4 \pm 3.8$ & $<0.001$ \\
\hline $\mathrm{HbAlc}$ & $7.2 \pm 1.1$ & $5.7 \pm 0.5$ & $<0.001$ & $7.0 \pm 1.0$ & $5.8 \pm 0.6$ & $<0.001$ & $7.3 \pm 1.2$ & $5.9 \pm 0.5$ & $<0.001$ \\
\hline FPI & $9.5 \pm 1.3$ & $8.5 \pm 0.7$ & $<0.001$ & $9.7 \pm 1.5$ & $8.8 \pm 0.6$ & $<0.001$ & $9.6 \pm 1.4$ & $8.7 \pm 0.8$ & 0.009 \\
\hline Systolic BP (mmHg) & $144.9 \pm 17.3$ & $106.6 \pm 10.5$ & $<0.001$ & $146.5 \pm 15.4$ & $105.8 \pm 10.7$ & $<0.001$ & $143.2 \pm 20.4$ & $105.4 \pm 9.7$ & $<0.001$ \\
\hline Diastolic BP (mmHg) & $102.3 \pm 15.9$ & $76.0 \pm 11.1$ & $<0.001$ & $101.9 \pm 15.4$ & $76.0 \pm 11.3$ & $<0.001$ & $103.5 \pm 16.5$ & $74.7 \pm 9.9$ & $<0.001$ \\
\hline T - Cholesterol (mg/dl) & $245.0 \pm 16.0$ & $156.9 \pm 21.2$ & $<0.001$ & $245.8 \pm 15.0$ & $149.2 \pm 17.9$ & $<0.001$ & $246.3 \pm 13.0$ & $149.6 \pm 9.3$ & $<0.001$ \\
\hline $\mathrm{HDL}-\mathrm{C}(\mathrm{mg} / \mathrm{dl})$ & $47.5 \pm 11.5$ & $46.6 \pm 9.2$ & 0.637 & $44.6 \pm 10.7$ & $45.7 \pm 8.7$ & 0.558 & $48.9 \pm 10.3$ & $46.4 \pm 7.9$ & 0.429 \\
\hline $\mathrm{LDL}-\mathrm{C}(\mathrm{mg} / \mathrm{dl})$ & $190.9 \pm 30.1$ & $110.3 \pm 22.2$ & $<0.001$ & $197.2 \pm 26.3$ & $103.5 \pm 19.2$ & $<0.001$ & $187.4 \pm 30.2$ & $103.1 \pm 11.9$ & $<0.001$ \\
\hline Triglycerides (mg/dl) & $355.9 \pm 95.9$ & $139.6 \pm 6.0$ & $<0.001$ & $357.0 \pm 98.8$ & $142.3 \pm 4.9$ & $<0.001$ & $365.8 \pm 109.7$ & $141.5 \pm 4.9$ & $<0.001$ \\
\hline
\end{tabular}

Notes: P-value < 0.05 considered significant, P-values are obtained from Univariate Analysis of Variance, Bonferroni correction has been applied, Data presented as Mean \pm SD. Abbreviations: BMI, body mass index; HbAlc, haemoglobin $\mathrm{A}_{\mathrm{Ic}}$; PPG, postprandial plasma glucose; HDL, high-density lipoprotein; WHR, waist-to-hip ratio; FPI, fasting plasma insulin; LDL, low-density lipoprotein; FPG, fasting plasma glucose.

LDL-C, triglycerides than controls. However, there was no significant difference between WHR and HDL-C.

Association between HHEX genotypes and investigated clinical characteristics are illustrated in Table 5. Subjects possessing GG, AG, and AA genotypes had significantly higher BMI, FPI, FPG, PPG, systolic BP, T - Cholesterol, HbA1c, diastolic BP, LDL-C, and triglycerides than controls. However, there was no significant difference among T2DM cases and healthy controls with genotypes GG, AG, and AA in WHR and HDL-C. Higher age was observed among T2DM cases with GG and AG genotypes in comparison with healthy individuals but not in subjects with AA genotypes.

Association between $\mathrm{CDKN} 2 \mathrm{~A} / 2 \mathrm{~B}$ genotypes and investigated clinical characteristics are presented in Table 6. With respect to the TT genotype, the levels of FPG, PPG, HbA1c, systolic BP, T-Cholesterol, diastolic $\mathrm{BP}, \mathrm{LDL}-\mathrm{C}$, and triglycerides were significantly higher in T2DM cases in comparison with healthy individuals, whereas insignificant age difference, BMI, WHR, FPI, and HDL-C. Compared with controls, T2DM subjects with TC and CC genotypes had significantly higher age, PPG, HbA1c, FPG, FPI, systolic BP, T - Cholesterol, BMI, diastolic BP, LDL-C, and triglycerides. However, we found no significant difference in WHR and HDL-C.

Association between IGF2BP2 genotypes and investigated clinical characteristics are shown in Table 7. With respect to TT genotype PPG, HbA1c, systolic BP, FPG, T-Cholesterol, diastolic BP, LDL-C, and triglycerides had significantly high values inpatient in comparison with controls, whereas did not find a significant difference among the values of age, BMI, WHR, FPI, and HDL-C. Except for WHR and HDL-C, all the other clinical parameters were significantly higher in T2DM people with GT and GG genotype in comparison with controls.

Effect of CDKAL1, HHEX, CDKN2A/2B, and IGF2BP2 polymorphism on HOMA- $\beta$ among T2DM subjects are shown in Table 8. HOMA- $\beta$ were compared with CDKAL1, HHEX, CDKN2A/2B, and IGF2BP2 genotypes, we observed no such statistically significant difference in HOMA- $\beta$ with respect to CDKAL1, HHEX, CDKN2A/2B, and IGF2BP2 genotypes. Higher HOMA- $\beta$ were observed with heterozygous and mutant genotypes of CDKAL1, HHEX, CDKN2A/2B compared to wild type genotypes while no such difference was observed with IGF2BP2 genotypes. 
Table 6 Clinical Characteristics Based on CDKN2A/2B Genotypes in T2DM Subjects and Controls

\begin{tabular}{|c|c|c|c|c|c|c|c|c|c|}
\hline \multirow[t]{2}{*}{ Parameters } & \multicolumn{3}{|l|}{ TT $(\mathrm{N}=44)$} & \multicolumn{3}{|l|}{$T C(N=168)$} & \multicolumn{3}{|l|}{$C C(N=257)$} \\
\hline & Disease & Control & P-values & Disease & Control & P-values & Disease & Control & P-values \\
\hline Age (Years) & $42.2 \pm 8.6$ & $41.0 \pm 9.0$ & 0.821 & $39.7 \pm 10.2$ & $35.2 \pm 7.5$ & 0.039 & $40.2 \pm 9.0$ & $35.1 \pm 8.1$ & $<0.001$ \\
\hline BMI (kg/m2) & $28.3 \pm 4.9$ & $24.7 \pm 2.2$ & 0.200 & $29.9 \pm 5.4$ & $24.6 \pm 2.5$ & $<0.001$ & $27.3 \pm 4.9$ & $24.9 \pm 2.3$ & $<0.001$ \\
\hline WHR & $0.9 \pm 0.1$ & $0.9 \pm 0.0$ & 0.474 & $0.9 \pm 0.0$ & $0.9 \pm 0.0$ & 0.750 & $0.9 \pm 0.1$ & $0.9 \pm 0.1$ & 0.557 \\
\hline FPG (mg/dl) & $145.3 \pm 27.2$ & $83.3 \pm 2.1$ & $<0.001$ & $133.3 \pm 25.0$ & $91.7 \pm 5.8$ & $<0.001$ & $134.1 \pm 26.1$ & $90.1 \pm 7.4$ & $<0.001$ \\
\hline PPG (mg/dl) & $216.2 \pm 39.3$ & $138.3 \pm 3.1$ & $<0.001$ & $201.4 \pm 35.1$ & $136.8 \pm 5.2$ & $<0.001$ & $203.8 \pm 35.4$ & $136.0 \pm 4.4$ & $<0.001$ \\
\hline $\mathrm{HbAlc}$ & $7.6 \pm 1.3$ & $6.0 \pm 0.0$ & 0.010 & $7.0 \pm 1.0$ & $5.9 \pm 0.5$ & $<0.001$ & $7.2 \pm 1.1$ & $5.7 \pm 0.5$ & $<0.001$ \\
\hline FPI & $9.5 \pm 1.3$ & $9.3 \pm 0.7$ & 0.749 & $9.5 \pm 1.4$ & $8.7 \pm 0.6$ & 0.010 & $9.6 \pm 1.4$ & $8.6 \pm 0.7$ & $<0.001$ \\
\hline Systolic BP (mmHg) & $144.5 \pm 19.4$ & $109.0 \pm 4.6$ & $<0.001$ & $144.9 \pm 17.3$ & $107.4 \pm 9.2$ & $<0.001$ & $145.4 \pm 16.9$ & $105.6 \pm 10.9$ & $<0.001$ \\
\hline Diastolic BP (mmHg) & $104.7 \pm 16.0$ & $70.7 \pm 10.2$ & $<0.001$ & $101.8 \pm 15.4$ & $79.6 \pm 11.2$ & $<0.001$ & $102.3 \pm 16.1$ & $75.1 \pm 10.8$ & $<0.001$ \\
\hline T-Cholesterol (mg/dl) & $243.9 \pm 15.4$ & $149.3 \pm 9.7$ & $<0.001$ & $243.5 \pm 16.0$ & $151.7 \pm 17.2$ & $<0.001$ & $247.5 \pm 14.2$ & $153.0 \pm 19.6$ & $<0.001$ \\
\hline $\mathrm{HDL}-\mathrm{C}(\mathrm{mg} / \mathrm{dl})$ & $48.8 \pm 8.9$ & $38.3 \pm 5.8$ & 0.098 & $44.7 \pm 10.2$ & $44.7 \pm 6.8$ & 0.995 & $48.1 \pm 12.1$ & $46.9 \pm 9.1$ & 0.419 \\
\hline LDL - C (mg/dl) & $182.1 \pm 35.0$ & $111.0 \pm 4.6$ & $<0.001$ & $195.3 \pm 26.0$ & $107.0 \pm 18.9$ & $<0.001$ & $192.1 \pm 29.7$ & $106.1 \pm 20.7$ & $<0.001$ \\
\hline Triglycerides (mg/dl) & $331.9 \pm 82.4$ & $143.7 \pm 3.8$ & $<0.001$ & $364.3 \pm 104.9$ & $14 \mid .5 \pm 4.7$ & $<0.001$ & $358.6 \pm 97.2$ & $140.8 \pm 5.8$ & $<0.001$ \\
\hline
\end{tabular}

Notes: P-value $<0.05$ considered significant, P-values are obtained from Univariate Analysis of Variance, Bonferroni correction has been applied, Data presented as Mean \pm SD. Abbreviations: BMI, body mass index; HbAlc, haemoglobin $\mathrm{A}_{\mathrm{Ic}}$ PPG, postprandial plasma glucose; HDL, high-density lipoprotein; WHR, waist-to-hip ratio; FPI, fasting plasma insulin; LDL, low-density lipoprotein; FPG, fasting plasma glucose.

\section{Discussion}

At present, over 150 different variants have been recognized by numerous studies, which are associated with T2DM. ${ }^{2,26}$ Environmental factors are linked with the onset of T2DM which involves a sedentary or inactive lifestyle, stress, and obesity. These risk factors do not influence similarly to everybody. ${ }^{27}$ We examined several clinicopathological parameters and a significant difference was found between T2DM cases and controls in BMI, FPG, HbA1c, PPG, FPI, systolic BP, Total-Cholesterol, diastolic BP, LDL - C, triglycerides, urination, fatigue, slow wound healing, appetite, alcohol consumption, weight loss, non-veg, smoking, consumption of fast food, blur vision, consumption of sugary drinks, excessive calorie intake, normal walk, brisk walk 20 minutes, prolonged sitting more than 3 hours work and leisure sitting tv mobile more than 3 hours whereas, no significant difference was found between T2DM cases and controls with respect to WHR, HDL - C, exercise, household chores, standing work more than 3 hours and family history.

Smoking, alcohol, consumption of sugary drinks, physical exercise, and family history are factors for the risk of T2DM. Several kinds of research have stated the negative impacts of smoking on T2DM. Smoking also enhances diabetes risk and intensifies the T2DM complications. ${ }^{28,29}$ In contrast, smoking cessation is linked with an increase in weight. Consumption of alcohol greatly influences the metabolism of lipids and can also affect the resistance of insulin and T2DM risk. $^{30}$ Consumption of sugary drinks was related with reduced concentration of HDL - C and bigger WHR ( $\mathrm{P}<0.001$, $\mathrm{P}=0.04$, respectively). ${ }^{31}$ A study confirmed the association of increasing consumption of sweet drinks and obesity and it is an important factor linked with the risk of $\mathrm{T}^{\mathrm{DDM}}{ }^{32}$ Exercise to fitness mortality association was observed in body compositions level. Among normalweight males with T2DM, mortality risk in the 1st quartile of fitness was $6.6(2.8-15.0)$, in the 2nd quartile was 3.2 $(1.4-7.0)$ and in the 3rd quartile was $2.2(1.1-4.6)$ in comparison with the 4 th quartile $(\mathrm{P}<0.0001) .{ }^{33}$ People with diabetes family history is a risk factor of T2DM and it is also linked with multiple metabolic disorders. ${ }^{34}$ However, in our analysis, we did not observe any relation of family history with T2DM. Many researchers suggested that biochemical parameters are highly associated with T2DM such as LDL-C and triglycerides but low level of 
Table 7 Clinical Characteristics Based on IGF2BP2 Genotypes in T2DM Subjects and Controls

\begin{tabular}{|c|c|c|c|c|c|c|c|c|c|}
\hline \multirow[t]{2}{*}{ Parameters } & \multicolumn{3}{|l|}{ TT $(\mathrm{N}=109)$} & \multicolumn{3}{|l|}{ GT $(N=136)$} & \multicolumn{3}{|l|}{ GG $(N=224)$} \\
\hline & Disease & Control & P-values & Disease & Control & P-values & Disease & Control & P-values \\
\hline Age (Years) & $39.8 \pm 8.8$ & $36.7 \pm 8.5$ & 0.331 & $39.9 \pm 9.4$ & $35.0 \pm 7.7$ & 0.011 & $40.7 \pm 9.9$ & $35.2 \pm 8.1$ & $<0.001$ \\
\hline BMI (kg/m2) & $26.7 \pm 5.0$ & $23.8 \pm 2.0$ & 0.054 & $31.8 \pm 4.2$ & $25.2 \pm 2.2$ & $<0.001$ & $27.3 \pm 5.0$ & $24.8 \pm 2.4$ & $<0.001$ \\
\hline WHR & $0.9 \pm 0.0$ & $0.9 \pm 0.1$ & 0.263 & $0.9 \pm 0.1$ & $0.9 \pm 0.0$ & 0.817 & $0.9 \pm 0.1$ & $0.9 \pm 0.0$ & 0.693 \\
\hline FPG (mg/dl) & $131.6 \pm 20.6$ & $90.9 \pm 7.5$ & $<0.001$ & $136.3 \pm 28.6$ & $89.9 \pm 7.1$ & $<0.001$ & $136.5 \pm 27.2$ & $90.3 \pm 7.2$ & $<0.001$ \\
\hline PPG (mg/dl) & $207.5 \pm 27.2$ & $138.4 \pm 4.1$ & $<0.001$ & $201.1 \pm 39.3$ & $135.4 \pm 5.4$ & $<0.001$ & $204.2 \pm 38.3$ & $136.3 \pm 4.0$ & $<0.001$ \\
\hline $\mathrm{HbAlc}$ & $7.0 \pm 1.0$ & $5.6 \pm 0.9$ & $<0.001$ & $7.1 \pm 1.1$ & $5.8 \pm 0.5$ & $<0.001$ & $7.3 \pm 1.2$ & $5.7 \pm 0.5$ & $<0.001$ \\
\hline FPI & $9.5 \pm 1.6$ & $8.8 \pm 0.9$ & 0.127 & $9.5 \pm 1.4$ & $8.7 \pm 0.8$ & 0.003 & $9.7 \pm 1.3$ & $8.6 \pm 0.6$ & $<0.001$ \\
\hline Systolic BP (mmHg) & $145.6 \pm 18.4$ & $109.2 \pm 10.9$ & $<0.001$ & $145.8 \pm 17.0$ & $105.1 \pm 9.7$ & $<0.001$ & $144.3 \pm 16.9$ & $106.0 \pm 10.7$ & $<0.001$ \\
\hline Diastolic BP (mmHg) & $100.7 \pm 16.0$ & $73.9 \pm 14.4$ & $<0.001$ & $102.6 \pm 15.7$ & $76.7 \pm 13.6$ & $<0.001$ & $103.3 \pm 15.7$ & $75.8 \pm 9.0$ & $<0.001$ \\
\hline T - Cholesterol (mg/dl) & $245.1 \pm 16.9$ & $148.1 \pm 15.6$ & $<0.001$ & $247.3 \pm 15.2$ & $154.1 \pm 18.6$ & $<0.001$ & $244.5 \pm 13.9$ & $152.6 \pm 19.5$ & $<0.001$ \\
\hline $\mathrm{HDL}-\mathrm{C}(\mathrm{mg} / \mathrm{dl})$ & $48.1 \pm 11.9$ & $44.3 \pm 4.6$ & 0.310 & $45.0 \pm 9.5$ & $46.1 \pm 9.9$ & 0.641 & $47.2 \pm 11.5$ & $46.5 \pm 8.6$ & 0.692 \\
\hline LDL - C (mg/dl) & $193.8 \pm 25.0$ & $103.8 \pm 16.3$ & $<0.001$ & $195.9 \pm 29.2$ & $108.0 \pm 21.2$ & $<0.001$ & $188.9 \pm 31.2$ & $106.0 \pm 20.0$ & $<0.001$ \\
\hline Triglycerides $(\mathrm{mg} / \mathrm{dl})$ & $393.7 \pm 106.2$ & $138.6 \pm 5.6$ & $<0.001$ & $358.0 \pm 98.6$ & $141.2 \pm 5.2$ & $<0.001$ & $335.8 \pm 88.5$ & $14 \mid .2 \pm 5.7$ & $<0.001$ \\
\hline
\end{tabular}

Notes: P-value < 0.05 considered significant, P-values are obtained from Univariate Analysis of Variance, Bonferroni correction has been applied, Data presented as Mean \pm SD. Abbreviations: BMI, body mass index; HbAlc, haemoglobin A $_{\text {c; }}$ PPG, postprandial plasma glucose; HDL, high-density lipoprotein; WHR, waist-to-hip ratio; FPI, fasting plasma insulin; LDL, low-density lipoprotein; FPG, fasting plasma glucose.

HDL - C. ${ }^{35}$ High consumption of sweet drinks containing a large amount of sugar increase the probability of being obese, especially in children. ${ }^{36}$ Hypertension is also related to T2DM risk. $^{37}$

Table 8 Comparison of HOMA- $\beta$ with Genotypes of CDKALI, HHEX, CDKN2A/2B, and IGF2BP2 in T2DM Subjects

\begin{tabular}{|c|c|c|c|c|}
\hline \multirow[t]{12}{*}{ HOMA- $\beta$} & \multicolumn{3}{|l|}{ CDKALI } & p-value \\
\hline & AA & $A C$ & $\mathrm{CC}$ & \multirow[t]{2}{*}{0.08} \\
\hline & $51.75 \pm 15.53$ & $58.80 \pm 42.72$ & $53.64 \pm 31.81$ & \\
\hline & \multicolumn{4}{|l|}{ HHEX } \\
\hline & GG & AG & AA & \multirow[t]{2}{*}{0.90} \\
\hline & $49.04 \pm 9.37$ & $55.24 \pm 31.98$ & $55.59 \pm 34.72$ & \\
\hline & \multicolumn{4}{|c|}{ CDKN2A/2B } \\
\hline & $\mathrm{CC}$ & $\mathrm{TC}$ & TT & \multirow[t]{2}{*}{0.09} \\
\hline & $45.82 \pm 12.56$ & $55.07 \pm 34.34$ & $88.81 \pm 29.87$ & \\
\hline & \multicolumn{4}{|l|}{ IGF2BP2 } \\
\hline & GG & TG & TT & \multirow[t]{2}{*}{0.21} \\
\hline & $53.90 \pm 25.7 \mid$ & $55.40 \pm 41.43$ & $53.90 \pm 25.30$ & \\
\hline
\end{tabular}

Notes: P-value $<0.05$ considered significant, Data presented as Mean \pm SD.
In the current research, we investigated the association of CDKAL1, HHEX, CDKN2A/2B, and IGF2BP2 gene polymorphism to T2DM risk among the Uttarakhand population. CDKAL1 gene plays an important part in beta cell dysfunction and susceptibility to T2DM. ${ }^{18}$ Association of SNP rs 10440833 in CDKAL1 with T2DM was found among Chinese ethnic groups. ${ }^{38,39}$ In our analysis, we observed CDKAL1 is significantly associated with T2DM risk. $\mathrm{C}$ allele's frequency was significantly high in T2DM cases in comparison with healthy individuals. The $\mathrm{c}$ allele was significantly found to increase the T2DM risk. Under the recessive model, CDKAL1 polymorphism exhibited significantly associated with T2DM risk. A similar finding was reported among the Han population of China. ${ }^{18}$ T2DM subjects with CC genotype had significantly higher WHR, FPG, PPG, HbA1c, systolic BP, T - Cholesterol, diastolic BP, LDL - C, and triglycerides compared to controls in the case of CDKAL1 gene. Previous studies found that CDKAL1 catalyzes the methyl group which can cause proinsulin misfolding and inhibiting the CDK5-p35 complex and hence, change the function of beta-cell and production of insulin. ${ }^{40}$ This indicates that CDKAL1 influence the insulin production and beta-cell function.

HHEX gene regulated beta-cell function and maturation by activating hepatocyte nuclear factor $-1 \mathrm{a} .{ }^{19}$ Association 
of HHEX SNP rs1111875 and T2DM was found significant in many Chinese studies. ${ }^{41,42}$ In the case of HHEX rs1111875, we observe that the genotype frequencies difference between T2DM cases and control were statistically non-significant. We did not find any significant difference in allelic frequency among T2DM cases and healthy controls. Similar results were reported by other studies among Indians. ${ }^{43,44}$ Our findings were inconsistent with the previous study by Bhowmick et al, among North-East Indian ethnic groups. This may be because of different ethnicity among different populations. ${ }^{19}$ We observed a significant association of HHEX gene polymorphism with T2DM only under the dominant model.

Association of rs10811661 SNP in CDKN2A/2B with T2DM has been confirmed by various GWAS in different populations. ${ }^{20-22}$ Our findings indicated a significant association of CDKN2A/2B rs10811661 gene polymorphism with T2DM. The frequency of both TC and TT genotype was higher among cases than control and associated with high T2DM risk. The frequency of CDKN2A/2B (rs10811661) T allele was significantly high in T2DM cases than in healthy individuals. Compared to the $\mathrm{C}$ allele, a significant increase in T2DM risk was documented with the $\mathrm{T}$ allele. Similar results were reported in Asian and European populations. $^{45-47}$ We found a significant association of CDKN2A/2B gene polymorphism with T2DM under the dominant model but did not find any significant association under the recessive model. Furthermore, with respect to TT genotype, the levels of FPG, PPG, HbA1c, systolic BP, T Cholesterol, diastolic BP, LDL - C, and triglycerides were significantly higher among T2DM cases than those in healthy individuals, whereas an insignificant difference was found in age, BMI, WHR, FPI and HDL - C. Compared with controls, T2DM subjects with TC and CC genotypes had significantly higher age, PPG, FPI, triglyceride, systolic BP, HbA1c, T Cholesterol, FPG, diastolic BP, LDL - C and BMI. WHR as well as HDL - $\mathrm{C}$ did not exhibit a significant association with T2DM risk in our analysis. In contrast, a study reported that WHR was significantly linked with CDKN2A/2B (rs10811661) risk allele among T2DM cases in the Chinese Han population with $\mathrm{p}=0.032 .{ }^{10} \mathrm{CDKN} 2 \mathrm{~A} / 2 \mathrm{~B}$ gene may influence the risk of T2DM by decreasing the expression and proliferation of beta-cell. $^{23}$

IGF2BP2 gene has been recognized to be linked with reduced secretion of insulin and it has a significant role in T2DM pathogenesis. ${ }^{25}$ GWAS found that genetic polymorphism in IGF2BP2 is linked with T2DM in various populations. ${ }^{48}$ We observed a significant association of
IGF2BP2 genetic variants with T2DM risk. We found that $\mathrm{T}$ allele frequency was higher in T2DM cases than in healthy individuals. We also observed that the TT genotype contributed to increased T2DM risk. Except for WHR and HDL-C, all the other clinical parameters were significantly higher in T2DM people with GT and GG genotype in comparison with controls. In addition, a significant relationship between IGF2BP2 rs4402960 G > T gene polymorphism and T2DM risk was found under the recessive and dominant model. Our findings were in accordance with a study conducted by Lyssenko et al, showing the association of recessive mutant genotype (TT) of IGF2BP2 polymorphism with increased T2DM risk. ${ }^{49}$ Similar findings were also observed in a meta-analysis among Indians, East Asians, and Caucasians. ${ }^{50}$ The limitation of the small sample size should be kept in mind. The validity of the inferences is worth confirming in a wider population study.

\section{Advantage versus Limitations of Study}

The study will help in unmasking the role of genes and biochemical parameters associated with type 2 diabetes. This will help in prevention and management of diabetes related complications. The main limitation of study is the unawareness and lack of information of disease. Late diagnosis of disease due to low literacy rate most of the people are not aware of disease and it primary symptoms.

\section{Conclusion}

Our study concluded that CDKAL1, CDKN2A/2B, and IGF2BP2 gene polymorphisms were significantly associated with increased risk of T2DM in the Uttarakhand population. In the case of HHEX, the genotype and allelic frequencies difference between T2DM cases and control were statistically non-significant. However, a significant association of HHEX gene polymorphism with T2DM was observed under the dominant model. The current study will be useful in the recognition of genetic risk factors linked to T2DM. More researches are required to further verify the association of these genes in the Indian population.

\section{Highlights}

- CDKAL1, CDKN2A/2B, and IGF2BP2 polymorphisms are associated with T2DM risk in the Uttarakhand population, India.

- HHEX polymorphism was statistically non-significant between T2DM cases and control. 
- The only dominant model of HHEX polymorphism shows a significant association with T2DM.

- Clinicopathological parameters like BMI, FPG, HbA1c, systolic BP increase T2DM risk among the population of Uttarakhand.

\section{Abbreviations}

T2D, type 2 diabetes; BMI, body mass index; IDF, International Diabetes Federation; PCOS, polycystic ovary syndrome; HMGA1, high-mobility group A1; TCF7L2, Transcription Factor 7 Like 2; CAPN10, Calpain-10; PPARG, Peroxisome Proliferator-Activated Receptor Gamma; KCNJ11, Potassium Inwardly Rectifying Channel Subfamily J Member 11; GWAS, Genome Wide Association Study; CDKAL1, CDK5 Regulatory Subunit Associated Protein 1-Like 1; HHEX, Haematopoietically Expressed Homeobox; CDKN2A/2B, Cyclin-Dependent Kinase Inhibitor 2A; IGF2BP2, Insulin Like Growth Factor 2 mRNA Binding Protein 2; SLC30A8, Solute Carrier Family 30 Member 8; MTNR1B, Melatonin Receptor 1b; KCNQ1, Potassium Voltage-Gated Channel, KQT-Like Subfamily, Member 1; WFS1, Wolframin ER Transmembrane Glycoprotein; HNF1B, Hepatocyte Nuclear Factor 1 Homeobox B; JAZF1, Juxtaposed with Another Zinc Finger Protein 1; CVD, cardiovascular Diseases; SNP, single nucleotide polymorphism; ANOVA, analysis of variance; PCR, polymerase chain reaction; RFLP, restriction fragment length polymorphism; FPG, fasting plasma glucose; FPI, fasting plasma insulin; PPG, postprandial glucose; LDL, lowdensity lipoprotein; HDL, high-density lipoprotein; Hbalc, haemoglobin $\mathrm{A}_{1 \mathrm{c}}$; $\mathrm{BP}$, blood pressure; OR, odds ratio; $\mathrm{CI}$, confidence interval; IEC, Institutional Ethics Committee; EDTA, ethylenediaminetetraacetic acid; ICMR, Indian Council Medical Research; GCP, Good Clinical Practice.

\section{Data and Material Availability Statement}

We confirm that the data used during the research will not be shared with anybody/broadcasted in any public domain. The datasets generated during and/or analysed during the current study are available from the corresponding author on reasonable request.

\section{Ethics Approval and Consent to Participate}

The study has been conducted only after the due clearance and approval from the Ethics committee of Jamia Millia
Islamia (vide Proposal No. 12/9/176/JMI/IEC/2018). As part of mandatory standardised ethical norm, a written informed consent was taken from the person before inclusion in the research work. The study conducted in accordance with the Declaration of Helsinki.

\section{Informed Consent}

Informed consent was obtained in writing from all participants prior to the study.

\section{Acknowledgments}

We gratefully acknowledge the Indian Council of Medical Research, New Delhi, India, and study subjects for their cooperation.

\section{Funding}

Indian council of medical research (ICMR), New Delhi provided funding for the study. (2017-3016/GENOMICS-BMS).

\section{Disclosure}

The authors report no conflicts of interest in this work.

\section{References}

1. IDF Diabetes Atlas, 9th edn. IDF Diabetes Atlas, 9th Edn. Brussels, and Belgium; 2019. Available from: https://www.diabetesatlas.org

2. Goyal Y, Verma AK, Joshi PC, Dev K. Contemplating the role of genetic variants of HHEX, CDKAL1, WFS1 and SLC30A8 genes of TYPE-2 diabetes in Asians ethnic groups. Gene Rep. 2019;17:100465. doi:10.1016/j.genrep.2019.100465

3. A. D. Association. Classification and diagnosis of diabetes: standards of medical care in diabetes-2020. Diabetes Care. 2020;43 (Supplement 1):S14-S31. doi:10.2337/dc20-S002.

4. Goyal Y, Verma A, Bhatt D, et al. Diabetes: perspective and challenges in modern era. Gene Rep. 2020;20:100759. doi:10.1016/j. genrep.2020.100759

5. Verma AK, Beg MMA, Saleem M, et al. Cell free TCF7L2 gene alteration and their association with Type 2 diabetes mellitus in North Indian population. Meta Gene. 2020;25:100727. doi:10.1016/j. mgene.2020.100727

6. International Diabetes Federation. IDF Diabetes Atlas 8th Edition. IDF Executive Office; 2017. Online Available from: http://www. diabetesatlas.org/

7. Mekala KC, Bertoni AG. Chapter 4 - Epidemiology of diabetes mellitus. In: Orlando G, Piemonti L, Ricordi C, Stratta RJ, Gruessner RWG, editors. Transplantation, Bioengineering, and Regeneration of the Endocrine Pancreas. Academic Press; 2020:49-58.

8. Zheng Y, Ley SH, Hu FB. Global aetiology and epidemiology of type 2 diabetes mellitus and its complications. Nat Rev Endocrinol. 2018;14(2):88-98. doi:10.1038/nrendo.2017.151

9. Chawla A, Chawla R, Jaggi S. Microvasular and macrovascular complications in diabetes mellitus: distinct or continuum? Indian J Endocrinol Metab. 2016;20(4):546-551. doi:10.4103/2230-8210.183480

10. Plengvidhya N, Chanprasert C, Chongjaroen N, Yenchitsomanus $P$, Homsanit M, Tangittipokin W. Impact of KCNQ1, CDKN2A/2B, CDKAL1, HHEX, MTNR1B, SLC30A8, TCF7L2, and UBE2E2 on risk of developing type 2 diabetes in Thai population. BMC Med Genet. 2018;19(1):93. doi:10.1186/s12881-018-0614-9 
11. Szabo M, Máté B, Csép K, Benedek T. Genetic approaches to the study of gene variants and their impact on the pathophysiology of type 2 diabetes. Biochem Genet. 2018;56(1-2):22-55. doi:10.1007/ s10528-017-9827-4

12. Verma AK, Beg MMA, Khan NA, Goyal Y, Dev K, Joshi PC. CDKAL1 ((rs10946398)) and TCF7L2 ((rs7903146)) gene polymorphisms and their association with risk of type-2 diabetes mellitus in population of Uttarakhand, India. Meta Gene. 2020;26:100767. doi:10.1016/j.mgene.2020.100767

13. Chiefari E. Functional variants of the HMGA1 gene and type 2 diabetes mellitus. JAMA. 2011;305(9):903. doi:10.1001/jama.2011.207

14. Verma A, Khan S, Joshi PC, Dev K. Study of risk aberrant of type-2 diabetes - current approaches for care and control. Int J Adv Res. 2016;4(12):2127-2129. doi:10.21474/IJAR01/2630

15. Adışen E, Uzun S, Erduran F, Gürer MA. Prevalence of smoking, alcohol consumption and metabolic syndrome in patients with psoriasis. An Bras Dermatol. 2018;93(2):205-211. doi:10.1590/ abd1806-4841.20186168

16. Walls ML, Hautala D, Gonzalez M, Greenfield B, Aronson BD, Onello E. Perceptions and prevalence of alcohol and cigarette use among american indian adults with type 2 diabetes. Clin Diabetes. 2019; cd180078. doi:10.2337/cd18-0078

17. Balducci S, D'Errico V, Haxhi J, et al. Level and correlates of physical activity and sedentary behavior in patients with type 2 diabetes: a cross-sectional analysis of the Italian diabetes and exercise study_2. PLoS One. 2017;12(3):e0173337. doi:10.1371/journal. pone. 0173337

18. Tian Y, Xu J, Huang T, et al. A novel polymorphism (rs35612982) in CDKAL1 is a risk factor of type 2 diabetes: a case-control study. Kidney Blood Press Res. 2019;44(6):1313-1326. doi:10.1159/000503175

19. Bhowmick A, Sarkar P, Baruah MP, et al. Association of SLC30A8, CDKAL1, TCF7L2 and HHEX gene polymorphisms with type 2 diabetes in the population of North East India. Cytol Genet. 2020;54(2):165-172. doi:10.3103/S0095452720020036

20. Voight BF, Scott LJ, Steinthorsdottir V, et al. Twelve type 2 diabetes susceptibility loci identified through large-scale association analysis. Nat Genet. 2010;42(7):579-589. doi:10.1038/ng.609

21. Saxena R, Voight BF, Lyssenko V, et al. Diabetes Genetics Initiative of Broad Institute of Harvard and MIT, Lund University, and Novartis Institutes of BioMedical Research. Genome-wide association analysis identifies loci for type 2 diabetes and triglyceride levels. Science. 2007;316(5829):1331-1336. doi:10.1126/science.1142358.

22. Steinthorsdottir V, Thorleifsson G, Reynisdottir I, et al. A variant in CDKAL1 influences insulin response and risk of type 2 diabetes. Nat Genet. 2007;39(6):770-775. doi:10.1038/ng2043

23. Kong Y, Sharma RB, Ly S, Stamateris RE, Jesdale WM, Alonso LC. CDKN2A/B T2D genome-wide association study risk SNPs impact locus gene expression and proliferation in human islets. Diabetes. 2018;67(5):872-884. doi:10.2337/db17-1055

24. Christiansen J, Kolte AM, Hansen TVO, Nielsen FC. IGF2 mRNA-binding protein 2: biological function and putative role in type 2 diabetes. J Mol Endocrinol. 2009;43(5):187-195. doi:10.1677/ JME-09-0016

25. Groenewoud MJ, Dekker JM, Fritsche A, et al. Variants of CDKAL1 and IGF2BP2 affect first-phase insulin secretion during hyperglycaemic clamps. Diabetologia. 2008;51(9):1659-1663. doi:10.1007/ s00125-008-1083-z

26. Suzuki K, Akiyama M, Ishigaki K, et al. Identification of 28 new susceptibility loci for type 2 diabetes in the Japanese population. Nat Genet. 2019;51(3):379. doi:10.1038/s41588-018-0332-4

27. Adeghate E, Schattner P, Dunn E. An update on the etiology and epidemiology of diabetes mellitus. Ann N Y Acad Sci. 2006;1084 (1):1-29. doi:10.1196/annals.1372.029

28. Jee SH, Foong AW, Hur NW, Samet JM. Smoking and risk for diabetes incidence and mortality in korean men and women. Diabetes Care. 2010;33(12):2567-2572. doi:10.2337/dc10-0261
29. Jebur H, Masroor M, Ahmad H, et al. CRP gene polymorphism and their risk association with type 2 diabetes mellitus. Open Access Maced J Med Sci. 2019;7(1):33-37. doi:10.3889/ oamjms.2019.014

30. Rimm EB, Williams P, Fosher K, Criqui M, Stampfer MJ. Moderate alcohol intake and lower risk of coronary heart disease: meta-analysis of effects on lipids and haemostatic factors. BMJ. 1999;319 (7224):1523-1528. doi:10.1136/bmj.319.7224.1523

31. Kosova EC, Auinger P, Bremer AA. The relationships between sugar-sweetened beverage intake and cardiometabolic markers in young children. J Acad Nutr Diet. 2013;113(2):219-227. doi:10. 1016/j.jand.2012.10.020

32. Lin W-T, Huang H-L, Huang M-C, et al. Effects on uric acid, body mass index and blood pressure in adolescents of consuming beverages sweetened with high-fructose corn syrup. Int $J$ Obes. 2013;37(4):532-539. doi:10.1038/ijo.2012.121

33. Church TS, Cheng YJ, Earnest CP, et al. Exercise capacity and body composition as predictors of mortality among men with diabetes. Diabetes Care. 2004;27(1):83-88. doi:10.2337/diacare.27.1.83

34. Meigs JB, Cupples LA, Wilson PW. Parental transmission of type 2 diabetes: the framingham offspring study. Diabetes. 2000;49 (12):2201-2207. doi:10.2337/diabetes.49.12.2201

35. Mullugeta Y, Chawla R, Kebede T, Worku Y. Dyslipidemia associated with poor glycemic control in type 2 diabetes mellitus and the protective effect of metformin supplementation. Indian $J$ Clin Biochem. 2012;27(4):363-369. doi:10.1007/s12291-012-0225-8

36. Darnton-Hill I, Nishida C, James WPT. A life course approach to diet, nutrition and the prevention of chronic diseases. Public Health Nutr. 2004;7(1a):101-121. doi:10.1079/PHN2003584

37. 'High blood pressure (hypertension) - Symptoms and causes', Mayo Clinic. Available from: https://www.mayoclinic.org/diseasesconditions/high-blood-pressure/symptoms-causes/syc-20373410. Accessed April 18, 2019.

38. Villegas R, Delahanty R, Gao Y-T, et al. Joint effect of genetic and lifestyle risk factors on type 2 diabetes risk among chinese men and women. PLoS One. 2012;7(11):e49464. doi:10.1371/journal. pone.0049464

39. Lu F, Qian Y, Li H, et al. Genetic variants on chromosome 6p21.1 and $6 \mathrm{p} 22.3$ are associated with type 2 diabetes risk: a case-control study in Han Chinese. J Hum Genet. 2012;57(5):320-325. doi:10.1038/jhg.2012.25

40. Kuo JZ, Sheu WH-H, Assimes TL, et al. Trans-ethnic fine mapping identifies a novel independent locus at the $3^{\prime}$ end of CDKAL1 and novel variants of several susceptibility loci for type 2 diabetes in a Han Chinese population. Diabetologia. 2013;56(12):2619-2628. doi:10.1007/s00125-013-3047-1

41. Qian Y, Lu F, Dong M, et al. Genetic Variants of IDE-KIF11-HHEX at $10 \mathrm{q} 23.33$ associated with type 2 diabetes risk: a fine-mapping study in Chinese population. PLoS One. 2012;7(4):e35060. doi:10.1371/journal.pone.0035060

42. Lu CC, Chen YT, Chen SY, et al. Hematopoietically expressed homeobox gene is associated with type 2 diabetes in $\mathrm{KK} \mathrm{Cg}$-Ay/J mice and a Taiwanese Han Chinese population. Exp Ther Med. 2018;16(1):185-191. doi:10.3892/etm.2018.6152

43. Chauhan G, Spurgeon CJ, Tabassum R, et al. Impact of common variants of PPARG, KCNJ11, TCF7L2, SLC30A8, HHEX, CDKN2A, IGF2BP2, and CD1KAL1 on the risk of type 2 diabetes in 5164 Indians. Diabetes. 2010;59(8):2068-2074. doi:10.2337/db09-1386

44. Kommoju UJ, Samy S, Maruda J, et al. Association of CDKAL1, CDKN2A/B \& HHEX gene polymorphisms with type 2 diabetes mellitus in the population of Hyderabad, India. Indian J Med Res. 2016;143(4):455. doi:10.4103/0971-5916.184303

45. Sakai K, Imamura M, Tanaka Y, et al. Replication study for the association of 9 East Asian GWAS-derived loci with susceptibility to type 2 diabetes in a Japanese population. PLoS One. 2013;8(9): e76317. doi:10.1371/journal.pone.0076317 
46. Binh TQ, Thu NTT, Phuong PT, Nhung BT, Nhung TTH. CDKN2Ars10811661 polymorphism, waist-hip ratio, systolic blood pressure, and dyslipidemia are the independent risk factors for prediabetes in a Vietnamese population. BMC Genet. 2015;16(1):107. doi:10.1186/ s12863-015-0266-0

47. Lee Y-H, Kang ES, Kim SH, et al. Association between polymorphisms in SLC30A8, HHEX, CDKN2A/B, IGF2BP2, FTO, WFS1, CDKAL1, KCNQ1 and type 2 diabetes in the Korean population. J Hum Genet. 2008;53(11-12):991-998. doi:10.1007/s10038-0080341-8
48. Koster JC, Marshall BA, Ensor N, Corbett JA, Nichols CG. Targeted overactivity of $\beta$ cell KATP channels induces profound neonatal diabetes. Cell. 2000;100(6):645-654. doi:10.1016/S0092-8674(00)80701-1

49. Lyssenko V, Jonsson A, Almgren P, Pulizzi N, Isomaa B, Tuomi T, Berglund G, Altshuler D, Nilsson P, Groop L. Clinical risk factors,

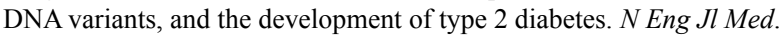
2008;359(21):2220-2232. doi:10.1056/NEJMoa0801869

50. Zhao Y, Ma YS, Fang Y, et al. IGF2BP2 genetic variation and type 2 diabetes: a global meta-analysis. DNA Cell Biol. 2012;31(5):713-720. doi:10.1089/dna.2011.1400

\section{Publish your work in this journal}

Diabetes, Metabolic Syndrome and Obesity: Targets and Therapy is an international, peer-reviewed open-access journal committed to the rapid publication of the latest laboratory and clinical findings in the fields of diabetes, metabolic syndrome and obesity research. Original research, review, case reports, hypothesis formation, expert opinion and commentaries are all considered for publication. The manuscript management system is completely online and includes a very quick and fair peer-review system, which is all easy to use. Visit http://www.dovepress.com/testimonials.php to read real quotes from published authors.

Submit your manuscript here: https://www.dovepress.com/diabetes-metabolic-syndrome-and-obesity-targets-and-therapy-journal 